\title{
Die Schriftheimat von Vat. Reg. Lat. 886 (Codex Theodosianus libri IX-XVI)
}

\author{
A.J.B. Sirks \\ University of Oxford \\ boudewijn.sirks@law.ox.ac.uk
}

\section{Summary}

It is generally assumed that the main manuscript of the Theodosian Code, Vat.Reg.Lat. 886 , was copied in the 6th century in South-East Gaul, although Italy as provenance is not excluded. This manuscript contains marginal summaries, of which the origin is also attributed to Gaul. However, it can be shown that the largest group was made by one of the scribes $\left(\mathrm{V}_{2}^{*}\right)$ after 535 and before 554 , on the very manuscript, that this was very likely done in Rome, and that the scribe was a Greek, perhaps a Byzantine official. This conclusion bears upon the provenance of Vat.Reg.Lat. 886. The errors in the Greek constitution CTh 9,45,4 imply that it cannot have been copied in the east. It must have been done in the west and not the Code, sent over in 437, was used (or else the Greek would be in order), but a copy of this Code, in which the scribe had misunderstood the Greek and made errors, which then figure in Vat.Reg.Lat. 886. The copying must have been done after 535 and just before the Summaria were made because the author of the Summaria was one of the correctors.

\section{Keywords}

Vat.Reg.Lat. 886 - Codex Theodosianus - Summaria Antiqua

1 Vat.Reg.Lat. 886 (v) ist eine Handschrift in der Vatikanischen Bibliothek. Sie wurde zuerst von Jean du Tillet erwähnt, der sie für seine Ausgabe des Codex Theodosianus von 155 o benutzte. Ihr Verbleib in jener Periode ist unbekannt. Paul Petau, der erst bekannte Eigentümer, hat sie möglicherweise von Daniel d'Orléans erworben, der sich 1562 mit der Bibliothek des geplünderten Klosters Fleury beschäftigt hat. Sie befand sich im 17. Jahrhundert in der 
Bibliothek des Sohnes Pauls, Alexandre Petau, der sie 1650 mittels Isaac Vossius an Christina von Schweden verkaufte. Nach Christinas Tode 1689 wurde sie mit ihrer Bibliothek noch im selben Jahr an die Vatikanische Bibliothek verkauft wo sie noch immer ist. Die Herkunft der Handschrift ist unsicher. Wegen der möglichen Verbindung mit Daniel d'Orléans vermutete Haenel, Fleury sei Heimatsort gewesen ${ }^{1}$. Das besagt aber nur, dass die Handschrift in irgendeinem Zeitpunkt dort gelangt sein könnte und nichts $\mathrm{mehr}^{2}$. Ebenso kann die Tatsache, dass das Breviarium Alarici, 506 für die Römer im westgotischen Königreich erlassen, auf dem Codex basiert und in Südfrankreich verbreitet war, kein Argument für eine Herkunft aus Südfrankreich sein: man kann eine andere Handschrift verwendet haben und es gibt nichts im Breviartext, das auf eine Herkunft aus dieser Handschrift weist. Ebensowenig weisen die Erweiterungen des Breviars im Buch 16. an hand eines Codex Theodosianus auf Vat.Reg. Lat. 886. Damit sind wir für die Herkunft des Codex auf die Merkmale der Handschrift angewiesen.

Die Handschrift umfasst die Bücher 9 bis 16, gebunden in einem Band. Sie ist von zwei Kopisten verfasst worden, Va und Vb. Diese zwei haben zwar einige Korrekturen angebracht, aber die meisten Korrekturen stammen von zwei anderen Personen, $\mathrm{V}_{2}{ }^{*}$ und $\mathrm{V}_{2}{ }^{3}$. Haenel meinte, die Schrift wäre nicht älter als das 7. Jahrhundert und entstamme dem Westen ${ }^{4}$. Mommsen war sich nicht sicher, woher die Handschrift genau kam (unde veniat, parum certum est). Er vermutete, auf Grund der Schrift, das sowohl Italien als Gallien Schriftheimat sein könnten. Gewisse spätere Marginalien waren merovingisch, was auf eine gallische Herkunft deuten würde. Obwohl das Griechisch genau so schön wie das Latein geschrieben war, und beide Kopisten beider Sprachen kundig waren, wies CTh 9,45,4, die einzige griechische Konstitution im Buch, nicht auf einen Mann hin, Graece doctus. Mommsen schrieb auch dem V2 die griechische Nummerierung der Quaternionen und gewisse griechische Noten $\mathrm{zu}^{5}$. Was dem Zeitpunkt des Entstehens anbelangte, Mommsen wollte diesen nicht

1 J.M. Coma Fort, Codex Theodosianus, Historia de un texto, Madrid 2014, S. 67, mit Verweis auf Haenel 1842, xxvii note 16oa, von Mommsen (infra, Fn. 2), S. XLV als unbegründet abgewiesen.

2 Coma Fort (supra, Fn. 1), S. S.71-72; Th. Mommsen, Theodosiani librixvI, adsumpto apparatu P. Krueger, Vol. I, pars prior, Prolegomena, Berlin 1905, S. XLV.

3 Mommsen (supra, Fn. 2), S. LVI schließt nicht aus, dass es um zwei Personen handelt, die einerseits V abgeschrieben haben, andererseits die Scholien hinzugefügt haben.

4 G. Haenel, Antiqua Summaria Codicis Theodosiani ex codice Vaticano nunc primum edita, Lipsiae 1834, S. XII.

5 Mommsen (supra, Fn. 2), S. LV: 'Ab eodem emendatore videntur proficisci tam numeratio quaternionum Graecis litteris ... quam numeri rubricis adiecti ...; für die Nummerierung und die Marginalien, siehe Mommsen (supra, Fn. 2), S. XLV und E.A. Lowe, Codices latini anti- 
früher als auf das 6. Jahrhundert (also nach 500) ansetzen ${ }^{6}$. Traube vermutete eine Herkunft aus Südfrankreich, möglicherweise Lyon ${ }^{7}$. Lowe datierte die Schrift auf das 6. Jahrhundert und vermutete ebenso Lyon als Herkunftsort. Dies, obwohl jeder Bogen griechisch nummeriert war und und jeder Bogen mit dem Chrismon anfängt, was man gewöhnlich in griechischen Handschriften findet. 'The manuscript is written with a pen cut for writing Greek, which lends a strange appearance to some Latin letters, but the Greek uncial found on foll. 75-76v [die griechische Version von CTh 9,45,4] shows pure Greek forms. ${ }^{8}$. Wie diese Merkmalen in Lyon im sechsten Jahrhundert in einer Handschrift zu erklären seien, sagt Lowe nicht. Sie deuten doch allerdings darauf, dass Va und $\mathrm{Vb}$ jedenfalls geübt im Schreiben von griechischen Texten waren und das dies ihre gewöhnliche Beschäftigung war. Wie sie dann im 6. Jahrhundert in Lyon eingetroffen sein könnten, bleibt rätselhaft und es spricht nicht für Lyon als Herkunftsort. Dass das Südosten Galliens und sogar Lyons als solches angenommen wird, basiert wahrscheinlich lediglich darauf, dass die Handschrift in Frankreich aufgetaucht ist und spätere merovingische Marginalien enthält. Das spricht jedenfalls für einen Verbleib in Gallien im 7. Jahrhundert (was mit der späteren Entdeckung durch D'Orléans übereinstimmt), aber der Codex könnte von anderswo in Gallien gelangt sein. Stolte zeigt sich skeptisch. Dass die griechische Version von CTh 9,45,5 von einer Person verfasst worden ist, die laut Mommsen des Griechischen unkundig war, bejaht Stolte insoweit, dass das Griechisch tatsächlich qualitativ sehr schlecht ist. Es lasse jedenfalls vermuten, dass der Codex nicht in Konstantinopel abgeschrieben wurde. Doch Stolte weist auch darauf hin, dass viele Irrtümer hier von einen unkundigen Kopisten falsch verstandenen griechischen Unzialen und somit von der Vorlage herrühren könnten. Auch er bezweifelt, ob sich die weiteren griechischen Merkmale mit Lyon als Schriftheimat vereinbaren lassen ${ }^{9}$. Salway ist ebenso

quiores, A palaeographical guide to Latin manuscripts prior to the ninth century, Oxford 1934, Vol. I, no. 110.

6 Th. Mommsen (supra, Fn. 2), S. XLVI, unter klarer Ablehnung von Haenels Behauptung, die Handschrift stamme aus Fleury.

7 Wie erwähnt von R.W.B. Salway, The publication of the Theodosian Code and transmission of its text, in: Société, économie et administration dans le Code Théodosien, edd. S. CrogiezPétrequin et P. Jailette, Lille 2012, S. 21-61, hier S. 25.

8 E.A. Lowe, Codices latini antiquiores, Vol. viII, Oxford 1959, nr. 110. R. Seider, Paläographie der Lateinischen Papyri, Band II,2, Stuttgart 1981, S. 89 meint: 'um 5oo n. Chr. in Südfrankreich, wahrscheinlich in Lyon, geschrieben', womit er sich Lowe anschliesst.

9 B.H. Stolte, The Use of Greek in the Theodosian Code, in: Subseciva Groningana 8, S. 147-159, hier S. 149-15o. Stolte nimmt an, S. 154, dass die Konstitution während des Editionsprozesses in den Titel aufgenommen wurde. Dass sie als separate Konstitution betrachtet wurde, wird auch bestätigt durch ihre Nummerierung als $v$. Aber der Summarist versah sie nicht mit 
skeptisch und meint, es lasse sich besser mit einer italienischen Herkunft vereinbaren, wofür er eine Handschrift, 517 in Verona angefertigt, heranzieht ${ }^{10}$. Beide Autoren waren richtig in ihrer Ablehnung von Lyon, wie wir sehen werden. Dickey weist darauf, dass der in den Text eingefügte Wortabbruch auf jemanden weist, der das Latein lernen musste ${ }^{11}$.

2 In der Handschrift sind zahlreiche Marginalien angebracht, die sogenannten Summaria Antiqua Codicis Theodosiani, lateinische Kurzfassungen des Inhalts einer Konstitution. Sie bilden zwei Gruppen. Eine, die bei weitem die größte Gruppe $S$, ist geschrieben von $\mathrm{V}_{2}{ }^{*}$, und eine, $\mathrm{S}_{2}$, geschrieben von $\mathrm{V}_{2}$, die nur vereinzelte Summarien umfasst ${ }^{12}$. Übernahme der Summarien der größten Gruppe aus einem anderen Codex kommt nicht in Frage, weil diese Summarien auf diesen Codex zusammengestellt sind. Es gibt in diesem Latein gewisse Verschreibungen die nur vom Schreiber $\mathrm{V}_{2}{ }^{*}$ stammen können. Eine Verschreibung im Summarium bei CTh 14,3,3 impliziert, dass $\mathrm{V}_{2}{ }^{*}$ der Autor der Summarien $S$ sein muss, während die Worttrennungen im Codex und die Summarien S2 nachher angebracht sind. Dabei ist Mommsens These, V2 hat einen anderen Codex verwendet für seine Summarien, nicht wahrscheinlich. Sie sind so lapidar, dass irgendjemand mit ausreichender Lateinkenntnisse sie auch hätte verfassen können ${ }^{13}$. Jedenfalls war der Summarist der größten Serie wahrscheinlich ein Grieche. Er kannte Latein, aber dieses Latein war soweit die Summarien das zeigen elementar ${ }^{14}$. Dazu kommt, dass das Summarium bei CTh 9,21,9, wo die Konstitution von falsae monetae rei quos paracharactas vocant spricht, qui parakapaximos faciunt hat, d.h., der Summarist verwendete

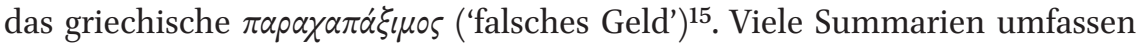

einem Summarium, was darauf hindeutet, dass er wusste, sie war mit der vorigen identisch; und was auch darauf deutet, dass der Summarist Griechisch kannte, sich darauf beschränkend eine Randnotiz hinzuzufügen: ' $\tau o ́$ $\pi \rho \sigma^{\sigma} \tau \alpha \gamma \mu \alpha$ ' (die Anordnung) (aber das Griechisch hier nicht korrigierte weil besonders die Bücher 9-12 nicht von ihm sondern von V2 korrigiert wurden: Mommsen (supra, Fn. 2), S. XVLI).

10 Salway (supra, Fn. 7), S. 25-27.

11 E. Dickey, Word division in bilingual texts, in: Signes dans les textes, textes sur les signes, eds G. Nocchi Macedo and M.C. Scappaticcio, Liège 2017, S. 159-175, here x 172-173. Sie vermutet, dass er Grieche war.

12 Mommsen (supra, Fn. 2), S. XLVI. Die Bezeichnungen sind Mommsens.

13 Siehe meinen Beitrag Summaria Antiqua Codicis Theodosiani, in REDHIS (Rediscovering the Hidden Structure), in Vorbereitung.

14 Mommsen (supra, Fn. 2), S. LI: 'Ipsorum scholiorum quae sit infantia et rerum ignorantia ... etc.'. D. Liebs, Die Jurisprudenz im spätantiken Italien (26o-640 n.Chr.), Berlin 1987, S. 182 ist freundlicher. Aber das Niveau würde übereinstimmen mit jenem der katapodas und mit einem byzantinischen Verwaltungsbediensteten.

15 Eine spätere Hand fügte in die Margine gegenüber als Erklärung: 'paracarac... falsos monetarios'. Siehe für die weiteren Merkmale meinen kommenden Beitrag (supra, Fn. 13). Siehe auch Fn. 9 . 
Querverweisen, oft mit der Bemerkung similis oder contraria, eine Praxis, die auch in c.Haec 2 erwähnt wird (resecatis tam supervacuis, quantum ad legum validitatem pertinet, praefationibus quam similibus et contrariis.

Manche Summarien sagen auch von einer Konstitution, sie sei inutilis, 'nutzlos', 'unbrauchbar' usw. ${ }^{16}$. Es stellt sich natürlich die Frage, warum sie nutzlos sei. In einem Beitrag über die Summaria in dieser Handschrift habe ich einige bisher unerklärliche Summarien anhand von einer Novelle Justinians gemeint deuten zu können. Es handelt sich um die Summarien zu CTh 15,8,1 und 2. Sie verbieten, kurz gesagt, Mädchen und Frauen zur Prostitution zu liefern. Der Summarist sagt von beiden, dass sie inutilis, veraltet sind. Gewiss ist das sonderbar weil dem Summaristen, der sich an anderer Stelle als orthodox Christen erweist (SCTh 16,5,37: a nostris katholicis), doch jedes Verbot von Prostitution teuer sein solle und nimmer nutzlos. Veraltet aber wäre möglich wenn es ein neues Verbot gab und das ist der Fall. Nicht im Westen, insoweit wir keine westliche Novelle bezüglich dieses Thema besitzen. Aber im Osten

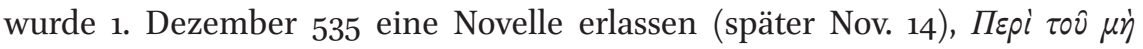

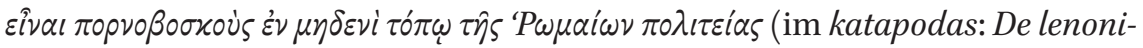
bus ) - 'Dass es keine Bordellhälter in irgendeinem Ort des römischen Staates gebe'. Hierin verbat Justinian jede Ausnützung von Prostituierten, d.h. die Prostitution, und damit, implizit, auch Frauenhandel wie in CTh 15,8,1 und 2 schon verboten ${ }^{17}$. Er veranlasste ausdrücklich, dass diese Novelle in allen Territorien unter seiner Gewalt veröffentlicht werden müsste, mit Inbegriff jener, ihm

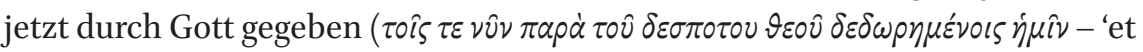
quae nunc a domino deo donata sunt nobis'). Dies schloss in jenem Zeitpunkt Africa und Sizilien ein (erobert 534 und 535) und Italien insoweit von Belisarius

16 K.E. Georges, Lateinisch-deutsches Handwörterbuch, Leipzig 1919, s.v. inutilis: I) negativ, nutzlos, unbrauchbar, unzuträglich, untauglich, ungeschickt; II) positiv, nachteilig, schädlich, hinderlich, verderblich, heillos.

17 CTh 15,8,1 verbietet den Verkauf von jungen Sklavinnen zur Prostitution wenn sie Christen sind. Nur Christen dürfen sie kaufen, wohl weil angenommen wird, sie werden sie nicht zur Prostitution aussetzen (hoffen wir, dass das auch der Fall war). CTh 15,8,2 verbietet Vätern und Eigentümern ihre Töchter bez. Sklavinnen zu prostituieren. Die Mädchen dürfen Bischöfe, Gouverneure und defensores um Hilfe bitten wenn sie fürchten, die Männer sind Zuhälter oder möchten sie prostituieren wollen. Den Männern wird dann ihre potestas bez. Eigentum entnommen und sie werden schwer bestraft. V2 ${ }^{*}$ sagt von CTh $14,8,1$, sie sei inutilis, nützlos, unanwendbar, und von 14,8,2, dass sie superiori similis sei. Tatsächlich ist c.2 insoweit similis, dass sie ebenso Prostitution bestreitet, aber c.1 handelt von Christenmädchen, c.2 betrifft alle Mädchen. C.1 handelt vom Verkauf zur Prostitution, c. 2 von Machtsmissbrauch. Der Unterschied ist im ursprünglichen Anwendungsbereich zu suchen: CTh 14,8,1 war für das Westen ausgefertigt, c.2 für das Osten. Damit betrafen sie verschiedene Fälle und verschiedene Bereiche. Es wundert deswegen nicht, dass CTh 14,8,2 in den Codex Justinianus von 534 aufgenommen wurde, durch die Novelle 14 von 535 aber wieder hintergestellt. Siehe weiter meinen kommenden Beitrag (supra, Fn. 13). 
Ende 535 erobert (Belisarius eroberte Rom 535 und erschlug Witigis 540). Hieraus folgt, dass der Summarist seine Summarien nach der Veröffentlichung dieser Novelle verfasst hat. Andere Summarien mit inutilis können auch mit dieser Novelle oder in Zusammenhang mit Novelle 105 von 537 erklärt werden ${ }^{18}$. Wieder andere Summarien weisen auf das Westen und zwar auf Rom als Ort der Verfassung. Der Summarist war sehr an der annona von Rom interessiert, und in SCTh 13,5,4, die von Getreideanfuhr aus Spanien handelt, muss das in hac urbe modo non tenet sich auf Rom beziehen, denn in Konstantinopel was man überhaupt nicht daran interessiert, in Rom dagegen wohl; aber in jener Zeit war Spanien nicht unter römischer Kontrolle ${ }^{19}$. Als Terminus ante quem gilt die Sanctio Pragmatica pro petitione Vigilii von 554, mit der die justinianische Gesetzgebung in Italien eingeführt wurde. Damit verlor der Codex Theodosianus in den byzantinischen Gebieten seine Geltung, aber nicht in Gallien und dem westgotischen Teil Spaniens.

Die zweite Gruppe Summarien, die gering ist, ist von V2 geschrieben und wahrscheinlich der Vorlage der Vat.Reg.Lat. 886 entnommen. Denn hätte es noch das Exemplar, 437 nach Rom geschickt, gegeben, wäre es, falls das Kopieren in Rom geschehen wäre, sehr wahrscheinlich verwendet worden und wäre das Griechisch von CTh 9,45,5 nicht so verstümmelt gewesen; und, falls zur Überprüfung sie herangezogen war, wäre, in Anbetracht dass die Schreiber sehr wahrscheinlich Griechen waren, CTh 9,45,5 sicherlich verbessert worden. Von Allem zeigt sich nichts (und müssen wir annehmen, das Exemplar, 438 dem Senat angeboten, war verschwunden). Aus Kreuzverweise (bei CTh 11,14,1 und 11,29,1 zu bez. Buch 1 und CTh. 1,24,1; und bei 12,16,1 zu Buch 4 mit den Titel De vectigalibus et commissis) ergibt sich, dass diese Vorlage vollständig war.

3 Wenn diese Annahme, die Summaria Antiqua seien zwischen 535 und 554 in Rom entstanden, richtig ist, hat sie Konsequenzen für die Handschrift Vat.Reg.Lat. 886 des Codex Theodosianus bezüglich ihrer Datierung und ihren Schriftheimat. Denn, wie Mommsen sagt, die Kopierarbeit von Va und $\mathrm{Vb}$ wurde von $\mathrm{V}_{2}{ }^{*}$ und $\mathrm{V}_{2}$ durchgenommen und korrigiert. Das geschah bevor

18 Gleicherweise können wir die Summarien bei CTh 15,7,10 (hodie vacat) und 11 (inutilis est) erklären. Sie handeln vom Verbot des Kaufes oder Verkaufes von fidicinae (Flötespielerinnen) und vom Verbot für mimae gewisse Juwelen und Kleider zu tragen. Byzantinern betrachteten alle weibliche Berufstätigen in diesem Bereich so gut wie Prostituierten, wie die Novelle 105.1 von 537 zeigt, wo der Aufzug zum Theater mit allerart Schauspielen rópval, 'Huren', genannt werden. Nach dem Erlass von Novelle 14 hatte das Verbot von CTh 15,7,10 tatsächlich seine Bedeutung verloren.

19 Also kommen andere Städte wie Syrakus nicht im Frage: dorthin gelangte nie regelmäßig Getreide aus Spanien. Siehe meinen kommenden Beitrag (supra, Fn. 13). 
sie ihre Summarien, die höchstwahrscheinlich in Rom verfasst wurden, niederschrieben. Va und $\mathrm{Vb}$ schrieben wie griechische Schreiber, waren also wahrscheinlich Byzantiner. Somit gab es eine enge Verbindung zwischen den Schreibern des Codex und den Schreibern der Summarien. Nach dem Kopieren des Codex wurde die größte Gruppe Summarien anhand des Codex verfasst und hierin durch $\mathrm{V}_{2}{ }^{*}$ niedergeschrieben. Danach kamen die Summarien der zweiten Gruppe, niedergeschrieben von V2. Der Summarist der größten Gruppe S war höchstwahrscheinlich Grieche, somit Byzantiner. Zeitlich können beide Arbeiten nicht lange aus einander gelegen haben. Es gibt dann zwei Möglichkeiten.

Va und Vb könnten in Konstantinopel den Codex kopiert haben, wonach $\mathrm{V}_{2}{ }^{*}$ und $\mathrm{V}_{2}$ ihre Arbeit kontrollierten, diese den Codex mitnahmen nach Rom und dort die Summarien, anhand des Codex dort verfasst, eintrugen. Wir hätten dann mit Vat.Reg.Lat. 886 die Kopie eines sich in Konstantinopel befindenden Codex, vielleicht sogar das Exemplar, für den Codex Justinianus benutzt. In diesem Falle wären die Verstümmelungen in der griechischen Version nicht erklärbar, wie von Stolte bemerkt. Somit fällt diese Möglichkeit aus.

Oder sowohl die zwei Schreiber Va und Vb und die zwei Schreiber V2* und $\mathrm{V} 2$ gingen mit der byzantinischen Verwaltung nach Rom. Va und Vb kopierten dort einen Codex und wurden am Ort von $\mathrm{V}_{2}{ }^{*}$ und $\mathrm{V}_{2}$ kontrolliert. Nach Mommsen hat $\mathrm{V}_{2}$ * für seine Emendationen sich nur von $\mathrm{V}$ bedient. Seine Emendationen sind immer Verschlechterungen. Dagegen waren die Emendationen von V2 immer Verbesserungen. Somit, so nimmt Mommsen an, hat V2 über ein besseres Exemplar des Theodosianus verfügt ${ }^{20}$. Aber auch $V_{2}$ hat in CTh 9,45,5 unrichtige Emendationen eingetragen und sein Exemplar war in diesem Punkt anscheinend nicht besser, wenn es überhaupt ein anderes Exemplar gegeben hat. Es kann weiter auch so sein, dass V2, zwar Grieche, über weit bessere Lateinkenntnisse verfügte als $\mathrm{V}_{2}{ }^{*}$ und für seine Emendationen keinen Codex brauchte sondern sich ebenso von V bediente. Hätte es sich dort das im 438 dem Senat überhändigte Exemplar gegeben, dann hätte es eine Chance gegeben, dass Verstümmelungen in der griechischen Fassung von CTh $9,45,5$ bemerkt und korrigiert worden waren. Weil das nicht ersichtlich ist, dürften die Verstümmelungen dann von einem früheren, italienischen Kopisten, der des Griechischen nicht mächtig war, herrühren, so wie Stolte schon andeutete. Das wiederum würde darauf hindeuten, dass das im Archiv verwahrte Exemplar, wovon die Gesta Senatus reden, nicht die Vorlage war, denn hier war das Griechisch zweifellos richtig. Vat.Reg.Lat. 886 muss dann in Rome 
zwischen 535 und 554 verfasst worden sein. $\mathrm{V}_{2}{ }^{*}$ und $\mathrm{V}_{2}$ trugen dann danach die Summarien ein.

4 Alles spricht somit dafür, dass der Codex Vat.Reg.Lat. 886 in Rom anhand eines dort sich befindenden Exemplar, einer Kopie des 437 nach dem Westen geschickten Exemplars, kopiert wurde, von griechischen Schreibern, in der byzantinischen Verwaltung tätig. Damit fallen Lyon und Südost-Gallien als Herkunftsort aus. Die Kontrolle von Va und $\mathrm{Vb}$ durch $\mathrm{V}_{2}{ }^{*}$ und $\mathrm{V}$ 2, die wieder die Summarien eintrugen, macht der Gedanke, die Abfassung und Eintragung der Summarien geschahen an verschiedenen Orten, unwahrscheinlich. Neben den inhaltlichen Indizien der Summaria spricht das für Rom als Schriftheimat des Codex. Die Datierung auf das 6. Jahrhundert wie von Mommsen und Lowe behauptet ist bestätigt. Dazu kommt, dass das Abfassen einer Kopie des Codex nur in der Periode 535-554 für Italien Bedeutung hatte ${ }^{21}$. Die Gräzismen und andere griechische Merkmale im Codex und in den Summarien deuten auf griechische Personen hin, das mangelhafte Latein ebenso auf Menschen, die nicht Latein als Muttersprache hatten, und die Verwendung von Worttrennungen im Codex, üblich bei denjenigen, die eine Fremdsprache lernen, deutet darauf, dass der Codex nachher von einem Griechen verwendet wurde; wohl in der byzantinischen Verwaltung Italiens. In dieser Periode oder nachher gelangte er im merovingischen Gallien, vielleicht über kirchlichen Verbindungen, da die gallische Kirche immer noch Interesse am Codex Theodosianus hatte, wie die Erweiterungen im 16. Buch des Breviars, genommen aus dem 16. Buch des Theodosianus, bezeugen.

$21 \quad$ Es ist nicht klar, ob die justinianische Kodifikation 534 mit der Eroberung Africas dort direkt eingeführt worden ist, oder erst später. CJ 1,27,1 datiert von 534, regelt aber nur die Verwaltung. 